\title{
Validation of post-traumatic stress disorder (PTSD) and complex PTSD using the International Trauma Questionnaire
}

Hyland P, Shevlin M, Brewin CR, Cloitre M, Downes AJ, Jumbe S, Karatzias T, Bisson JI, Roberts NP. Validation of post-traumatic stress disorder (PTSD) and complex PTSD using the International Trauma Questionnaire.

Objective: The 11th version of the International Classification of Diseases (ICD-11) has proposed two related trauma diagnoses: Posttraumatic stress disorder (PTSD) and Complex PTSD (CPTSD). Using a newly developed, disorder-specific measure of PTSD and CPTSD called the International Trauma Questionnaire (ITQ) the current study will (i) assess the factorial validity of ICD-11 PTSD and CPTSD; (ii) provide the first test of the discriminant validity of these constructs; and (iii) provide the first comparison of ICD-11, and Diagnostic and Statistical Manual, Fifth Edition (DSM-5), PTSD diagnostic rates using disorder-specific measures.

Method: ICD-11 and DSM-5 PTSD-specific measures were completed by a British clinical sample of trauma-exposed patients $(N=171)$. The structure and validity of ICD-11 PTSD and CPTSD were assessed by means of factor analysis and assessing relationships with criterion variables.

Results: Diagnostic rates under ICD-11 were significantly lower than those under DSM-5. A two-factor second-order model reflecting the distinction between PTSD and CPTSD best represented the data from the ITQ; and the PTSD and CPTSD factors differentially predicted multiple psychological variables.

Conclusion: The factorial and discriminant validity of ICD-11 PTSD and CPTSD was supported, and ICD-11 produces fewer diagnostic cases than DSM-5.

\author{
P. Hyland ${ }^{1,2}$ iD, M. Shevlin ${ }^{3}$, \\ C. R. Brewin ${ }^{4}$, M. Cloitre ${ }^{5,6}$, \\ A. J. Downes ${ }^{7}$, S. Jumbe ${ }^{8}$, \\ T. Karatzias ${ }^{9,10}$ (D), J. I. Bisson ${ }^{11, *}$, \\ N. P. Roberts ${ }^{12, *}$ \\ ${ }^{1}$ National College of Ireland, ${ }^{2}$ Centre for Global Health, \\ Trinity College Dublin, Dublin, Ireland, ${ }^{3}$ School of \\ Psychology, Ulster University, Derry, ${ }^{4}$ Clinical \\ Educational \& Health Psychology, University College \\ London, London, UK, ${ }^{5}$ School of Medicine, New York \\ University, New York, NY, ${ }^{6}$ National Center for PTSD, \\ Veterans Affairs Palo Alto Health Care System, Palo \\ Alto, CA, USA, ${ }^{7}$ St Mark's Dee View Surgery, Betsi \\ Cadwaldr Health Board, Connah's Quay, ${ }^{8}$ Centre for \\ Primary Care and Public Health, Queen Mary University \\ of London, Research Design Service London, London, \\ ${ }^{9}$ School of Health \& Social Care, Edinburgh Napier \\ University, ${ }^{10}$ Rivers Centre for Traumatic Stress, NHS \\ Lothian, Edinburgh, ${ }^{11}$ School of Medicine, Cardiff \\ University, and ${ }^{12}$ Cardiff \& Vale University Health \\ Board, Cardiff, UK
}

Key words: post-traumatic stress disorder; complex posttraumatic stress disorder; ICD-11; DSM-5; stress-related disorders; anxiety and depression

Philip Hyland, National College of Ireland, International Financial Services Center, Mayor Street, Dublin 1, Ireland.

E-mail: philip.hyland@ncirl.ie

*Jonathan I. Bisson and Neil P. Roberts are joint senior authors.

Accepted for publication June 19, 2017

\section{Significant outcomes}

- Diagnostic rates of ICD-11 PTSD and CPTSD are significantly lower than DSM-5 PTSD.

- The factorial validity of ICD-11 PTSD and CPTSD was supported using the newly developed International Trauma Questionnaire.

- The discriminant validity of ICD-11 PTSD and CPTSD was evidenced for the first time.

\section{Limitations}

- This study was based on a relatively small clinical sample, limiting generalizability of findings.

- Diagnostic rates for ICD-11 and DSM-5 were estimated without a measure of functional impairment.

- The ITQ is still under development and will be reduced in length in the near future to simplify the calculations required to meet the ICD-11 diagnoses for PTSD and CPTSD. 


\section{Hyland et al.}

\section{Introduction}

The forthcoming International Classification of Diseases version 11 (ICD-11) will include a diagnosis of Post-traumatic stress disorder (PTSD) comprised of six symptoms reflecting three factors: (i) Re-experiencing in the here and now (Re: two symptoms), (ii) deliberate avoidance (Av: two symptoms), and (iii) a sense of current threat (Th: two symptoms) (1). A sibling diagnosis, Complex PTSD (CPTSD), will also be included in ICD-11 and will be comprised of the six PTSD symptoms plus an additional set of symptoms that reflect 'Disturbances in Self-Organization' (DSO). These DSO symptoms are intended to capture the pervasive psychological disturbances that can occur following exposure to trauma, particularly those of an interpersonal nature that occur in early development, that are of a repeated and prolonged nature, and from which escape is difficult or impossible. The ICD-11 Working Group for Disorders Specifically Associated with Stress have not yet finalized the number of DSO symptoms that will be included in ICD-11; however, the DSO symptoms will comprise three factors: (i) Affective Dysregulation (AD), (ii) Negative Self-Concept (NSC), and (iii) Disturbed Relationships (DR) (1). Given their symptom composition, PTSD is conceptualized as a fear-based disorder, whereas CPTSD is conceptualized a broader clinical disorder that characterizes the impact of trauma on emotion regulation, identity, and interpersonal domains.

Several studies have sought to compare diagnostic rates of ICD-11 PTSD and CPTSD to DSM-5 PTSD. Findings have suggested a general trend for the ICD-11 to generate significantly lower diagnostic rates compared with the DSM-5 (2-4). In addition, considerable support for the factorial validity of ICD-11 PTSD (2-5) and CPTSD (6-9) has accrued. These studies are limited; however, as each relied on the use of proxy variables derived from secondary data sources to capture the ICD11 PTSD and DSO symptoms. To have a standardized assessment of these symptoms which aligns with the ICD-11 diagnostic criteria, the International Trauma Questionnaire (ITQ) (10) was developed. An initial confirmatory factor analytic (CFA) study amongst a clinical sample indicated that the latent structure of the ITQ was consistent with the proposed two-factor second-order model of CPTSD (i.e., a conceptual model that distinguishes between PTSD and CPTSD symptoms) (11). Additionally, mixture-modeling studies have supported the presence of distinct classes of trauma survivors characterized by PTSD and CPTSD symptom profiles $(12,13)$.
Aims of the study

Given the recent development of the ITQ, and the limited number of studies which have tested the validity of the PTSD and CPTSD proposals using this measure, the current study was performed to address two primary goals. First, we sought to determine whether the factor structure of the ITQ reported by Karatzias et al. (11) could be replicated amongst a distinct clinical sample (factorial validity). Second, we sought to extend upon existing knowledge by (i) performing the first comparison of ICD-11 PTSD/CPTSD diagnostic rates and DSM-5 PTSD diagnostic rates using disorderspecific measures (the ITQ and the PCL-5 (14)); and (ii) to provide the first assessment of the discriminant validity of ICD-11 PTSD and CPTSD. Three hypotheses were formulated in line with theoretical proposals and previous empirical findings. First, based on existing data (2-4), it was hypothesized that fewer people would meet diagnostic status under ICD-11 (PTSD and CPTSD) than DSM-5 (PTSD). Second, in line with theoretical proposals (1) and empirical findings (11), it was hypothesized that factorial models of the ITQ which discriminate between PTSD and DSO symptoms would offer optimal model fit. Third, consistent with the distinct symptom compositions of ICD-11 PTSD (fear/anxiety-based symptoms elicited by trauma-related cues) and CPTSD (emotional regulatory, self-conceptual, and interpersonal symptoms which are pervasive and not bound to trauma-related stimuli), it was hypothesized that the disorders would be differentially associated with six criterion variables. Specifically, it was hypothesized that the PTSD factor(s) would be stronger predictors of panic disorder (PD) and generalized anxiety disorder (GAD) symptoms than the CPTSD factor(s); and the CPTSD factor (s) would be stronger predictors of symptoms of major depressive disorder (MDD), negative trauma-cognitions, and distress tolerance than the PTSD factor(s).

\section{Methods}

Participants and procedures

Participants ( $n=171 ; 51.5 \%$ male) were recruited via the National Centre for Mental Health (http:// www.ncmh.info/). Participants were mental health service users who were recruited to $\mathrm{NCMH}$ via primary and secondary mental health services, specialist veteran's services, a specialist civilian trauma service, and via social media. Participants were eligible for the study if they were aged 18 or 
older and they reported that they had previously been given a diagnosis of PTSD, or if they indicated exposure to a traumatic event and screened positively for PTSD on the basis of the Trauma Screening Questionnaire. Exclusion criteria included inability read and write in English or disturbed mental state, requiring recent admission to hospital or intensive home treatment. All participants reported exposure to a traumatic event fulfilling the gateway criterion for a diagnosis of PTSD and CPTSD under ICD-11 and DSM-5. Participants ranged in age from 18 to 78 years $(M=49.85, S D=12.73)$ were primarily Caucasian $(n=162,95.9 \%), \quad$ and unemployed $(n=113,66.9 \%)$. Most were married or cohabiting ( $n=84,49.7 \%)$, while the remainder were single $(n=35,20.7 \%)$, divorced/separated $(n=43$, $25.5 \%)$, or widowed $(n=7,4.1 \%)$. A small proportion did not finish school $(n=12,7.1 \%)$, and many had attended higher education $(n=80$, $47.6 \%$ ). This study received ethical approval from the United Kingdom's National Research Ethics Service.

\section{Measures}

Traumatic exposure. A modified version of the Life Events Checklist for DSM-5 (LEC-5) (15) was used to assess lifetime exposure to traumatic events (with two additional items that assessed exposure to childhood physical abuse, and childhood sexual abuse, or molestation). Individuals were deemed to have been exposed to a trauma if they reported that an event 'Happened to me' or 'Witnessed it happening to somebody else.' A summed total score of types of trauma exposure was computed with a range of possible scores from 0 to 19 .

ICD-11 PTSD and DSO symptoms. The International Trauma Questionnaire (ITQ, version 1.2) (10) is a preliminary-stage self-report measure of the ICD-11 diagnoses of PTSD and CPTSD. This version of the scale contains a larger pool of items than will be included in the final version which will correspond to the ICD-11 diagnostic rules when finalized by the World Health Organization. Six items measure three PTSD clusters: (i) Re-experiencing in the here and now (Re1, Re2); deliberate avoidance of traumatic reminders (internal or external) (Av1, Av2); and a sense of current threat (Th1, Th2). A third Re item (Re3: Feeling very upset when something reminded you of the experience) was also included. Although it is non-specific in the sense that it does not address the ICD-11 concept of re-experiencing in the here and now, it is currently under consideration for use with traumatized individuals who possess no clear memory of their index trauma (e.g., possibly due to childhood traumatization or traumatic brain injuries). ${ }^{a}$ Sixteen items measure the three DSO factors: (i) Affective dysregulation, both hyperactivation (AD1-AD5) and hypoactivation (AD6AD9); (ii) negative self-concept (NSC1-NCS4); and (iii) disturbances in relationships (DR1-DR3).

Respondents are instructed to answer the PTSD question in relation to how much they have been bothered by each symptom in the past month, and are instructed to answer the DSO items in relation to how they typically feel, think about themselves, and relate to others. All items are answered on a five-point Likert scale anchored by 'Not at all' (0) and 'Extremely' (4). Diagnostic criteria for PTSD require a score of $\geq 2$ ('Moderately') for at least one of two symptoms from the Re, Av, and Th clusters. CPTSD diagnosis requires that the PTSD criteria are met and endorsement of each DSO symptom cluster at a moderate level of severity, defined as summed score that equals a score of $\geq 2$ for each of the items in the cluster): a summed total score of $\geq 10$ for items AD1-AD5 (reflecting hyperactivation) or a summed total score of $\geq 8$ for items AD6-AD9 (reflecting hypoactivation); a summed total score $\geq 8$ for items NSC1-NSC4; and a summed total score $\geq 6$ for items DR1-DR3. The ICD-11 requires the presence of functional impairment associated with both sets of symptoms for a diagnosis of PTSD and CPTSD. However, functional impairment was not assessed in the current study; therefore, diagnostic rates are based on symptom criteria alone. The ICD-11's taxonomic structure means that an individual can only be diagnosed with PTSD or CPTSD, not both.

DSM-5 PTSD symptoms. The PTSD Checklist for DSM-5 (PCL-5) (14) is a 20-item, self-report measure of intrusions (I: 5 items), avoidance (Av: 2 items), negative alterations in cognition and mood (NACM: 7 items), and alterations in arousal and reactivity (Ar: 6 items). The PCL-5 uses the same Likert scale response format as the ICD-TQ, and respondents answer each question in relation to how much they have been bothered by a symptom in the past month. PTSD diagnosis can be made if an individual reports a score of $\geq 2$ for at least one I symptom, at least one Av symptom, at least two NACM symptoms, and at least two Ar symptoms. As with ICD-11, DSM-5 requires endorsement of functional impairment for diagnosis; however, this

\footnotetext{
${ }^{\text {a }}$ Please note that Re3 is currently considered as a test item for diagnostic purposes and thus will not be included in any of the modeling analyzes in this study.
} 


\section{Hyland et al.}

was not assessed. Diagnostic rates are thus based on symptom criteria alone. Studies have reported acceptable psychometric properties for the PCL-5 (16). Reliability estimates among the current sample were satisfactory: I $(\alpha=.90), \operatorname{Av}(\alpha=.87)$, $\operatorname{NACM}(\alpha=.84)$, and $\operatorname{Ar}(\alpha=.78)$.

Criterion variables

The GAD-7 (17) is a seven-item measure of DSMIV GAD. Respondents indicate how much they are bothered by each symptom over the past 2 weeks. Each item is scored on a three-point Likert scale $(1=$ 'not at all', $3=$ 'more than half the days'). The GAD-7 has demonstrated good psychometric properties among clinical samples (18), and the reliability of the scale among the current sample was satisfactory $(\alpha=.86)$.

The PHQ-9 (19) is a nine-item measure of DSMIV major depressive disorder. Respondents indicate how much they are bothered by each symptom over the past two weeks on a four-point Likert scale $(0=$ 'not at all', $3=$ "nearly every $\left.d a y^{\prime}\right)$. The PHQ-9 possesses satisfactory psychometric properties (20), and the reliability among the current sample was acceptable $(\alpha=.89)$.

The PHQ-Panic Disorder (PHQ-PD) (21) is a 15-item measure of PD derived from the DSM-IV criteria. The first four questions (3a-3d) screen for whether a person has experienced an anxiety attack in the past 4 weeks. The remaining 11 questions $(4 \mathrm{a}-4 \mathrm{k})$ measure somatic (e.g., heart palpitation) and psychological (e.g., afraid of dying) symptoms. Each symptom is answered on a 'yes' (1) or 'no' (0) basis. A summed total score of panic symptoms is based on responses to the 11 symptomatic questions. PHQ-PD scores possess satisfactory psychometric properties (21), and the reliability amongst the current sample was good $(\alpha=.91)$.

The Post-traumatic Cognitions Inventory (PTCI) (22) contains 33 self-report items which measure negative beliefs about the self ('Self': 21 items), the world ('World': 7 items), and self-blame (5 items). For the current study, the Self and World subscales were selected. Items are scored on a seven-point Likert scale ( $1=$ 'totally disagree,' $7=$ 'totally agree') and higher scores reflect increasingly negative beliefs. The reliability of the Self $(\alpha=.95)$ and World $(\alpha=.90)$ subscales were good.

The Distress Tolerance Scale (23) is a 16-item self-report measure of one's ability to regulate emotions, accept distressing emotions, and function effectively when distressed. Items are scored on a five-point Likert scale ( 1 = 'strongly disagree,'
$5=$ 'strongly agree'), and higher scores reflect higher levels of distress tolerance. The scale possesses good psychometric properties (24), and the internal reliability among the current sample was satisfactory $(\alpha=.87)$.

\section{Data analysis}

Combined ICD-11 PTSD and CPTSD diagnostic rates were compared to DSM-5 PTSD diagnostic rates using the $z$-test. Diagnostic agreement between the two systems was assessed using Cohen's kappa statistic where a value $>.61$ indicates acceptable agreement (25). The fit of seven alternative factor models of the ITQ, as outlined by Shevlin et al. (9) and Karatzias et al. (11) (see Fig. 1), were investigated using CFA in Mplus 7.4 (26). The mean and variance-adjusted weighted least squares (WLSMV) estimator was used as it provides accurate parameter estimates, standard errors, and test-statistics for ordinal indicators (27). Missing data were managed using the pairwise present analysis method. Acceptable model fit was indicated by a chisquare-to-degree of freedom ratio of less than 3:1 (28); CFI and TLI values $>.90$ indicate adequate fit, and values $>.95$ indicate excellent fit (29); and RMSEA values $<.08$ indicate adequate fit, and values <.06 indicate excellent fit (30). The WLSMV estimator does not produce information-based indices; thus, the models were also fitted using robust maximum likelihood (MLR) (31) estimation to generate the Bayesian Information Criterion (BIC). The BIC is used to compare nested and non-nested models, and the model with the smaller value is deemed to be a better-fitting model. A 6- to 10-point difference between two models indicates strong evidence that the model with the lower value is statistically superior (32).

Upon selection of the best fitting model, composite reliability analysis was performed to determine the internal reliability of the ITQ. Composite reliability calculates internal consistency without the assumption of tau equivalence and thus is more appropriate for measures with small numbers of items, such as the ITQ. Values $>.60$ indicate acceptable internal reliability (33).

Finally, summed PTSD/DSO scores based on the best fitting model of the ITQ were entered into a hierarchical multiple regression model to predict six criterion variables. Gender, age, employment status $(0=$ employed, $1=$ unemployed $)$, and relationship status $(0=$ in a relationship, $1=$ not in a relationship) were entered at Step 1, and the PTSD and DSO variables were entered at Step 2. 


\section{Results}

Descriptive statistics and diagnostic estimates

The mean number of types of traumatic life events experienced was $6.75(M d n=6.00, S D=3.84)$, and the most frequently experienced trauma was physical assault $(n=121,73.7 \%)$. The experience of physical and/or sexual abuse during childhood was common $(n=79,47.6 \%)$. The most distressing traumatic events identified were childhood sexual abuse or molestation $(n=26,15.5 \%)$, and combat $(n=22,13.1 \%)$. Most respondents indicated a clear memory of their index (worst) trauma ( $n=144,87.8 \%)$. Amongst those who did not, the most common events associated with no memory were childhood sexual abuse or molestation (35\%, $n=7)$ and physical assault (20\%, $n=4)$.

The probable diagnostic rate for ICD-11 PTSD and CPTSD was $64.5 \%(n=107)$. Following the ICD-11's guidelines that a person may only receive a diagnosis of PTSD or CPTSD, but not both, it was necessary to calculate diagnostic rates for each diagnosis separately. The probable PTSD diagnostic rate was $10.9 \% \quad(n=18)$, and the probable CPTSD diagnostic rate was 53.6\% $(n=89)$. With
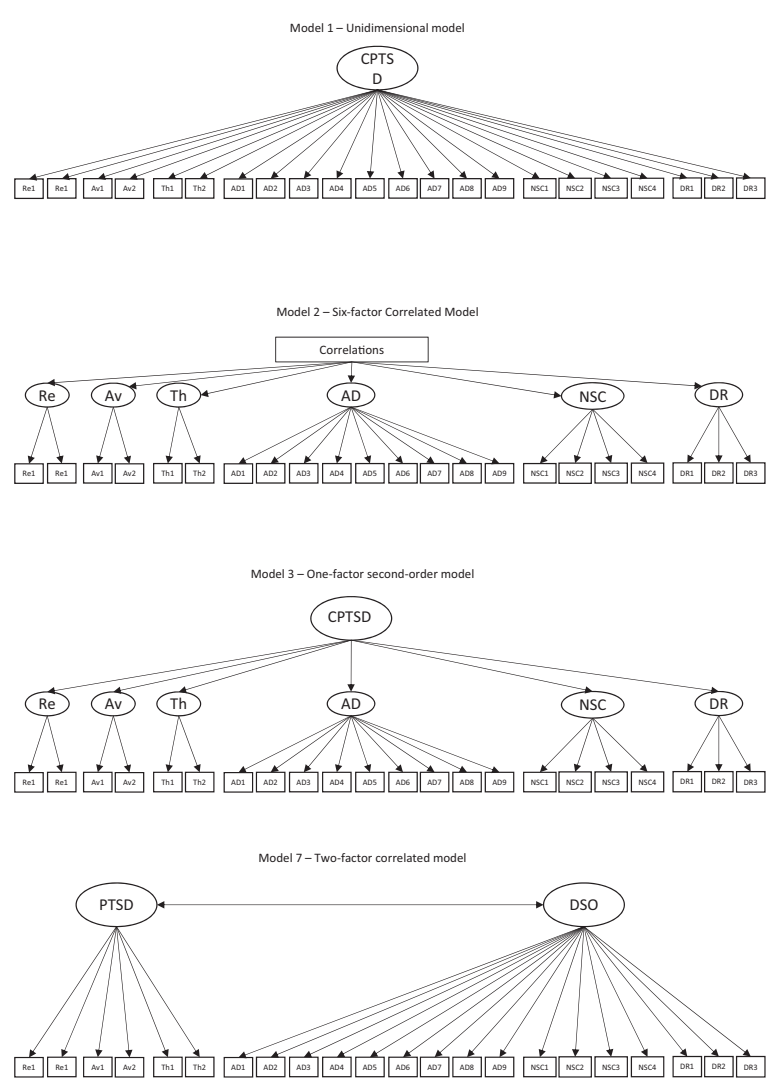

the inclusion of Re3, the combined PTSD and CPTSD probable diagnostic rate increased to $71.7 \%(n=119)$; with PTSD increasing to $12.7 \%$ $(n=21)$, and CPTSD increasing to $59.0 \%$ $(n=98)$. Endorsement rates for each ITQ item, based on one's diagnosis (PTSD or CPTSD), are displayed in Table 1.

The DSM-5 PTSD probable diagnostic rate was $76.1 \% \quad(n=124)$; a diagnostic rate significantly higher than the combined ICD-11 PTSD and CPTSD rate $(76.1 \%$ vs. $64.5 \%, z=2.30, \mathrm{SE}=.05$, $P=.01$ ). One hundred and three (of 163) participants shared an ICD-11 and DSM-5 diagnosis; 21 participants received a diagnosis under DSM-5 but did not receive a diagnosis under ICD-11, and one person received a diagnosis under ICD-11 but did not receive a diagnosis under DSM-5. This level of diagnostic agreement between the two systems was considered to be reasonably high (Kappa $=.69$, $\mathrm{SE}=.06, P<.001)$.

The DSM-5 PTSD diagnostic rate was not significantly different from the combined ICD-11 PTSD and CPTSD rate when the non-specific Re3 symptom was included within the ICD-11 diagnostic algorithm $(76.1 \%$ vs. $71.7 \%, \quad z=0.97$, $\mathrm{SE}=.05, P=.18)$.
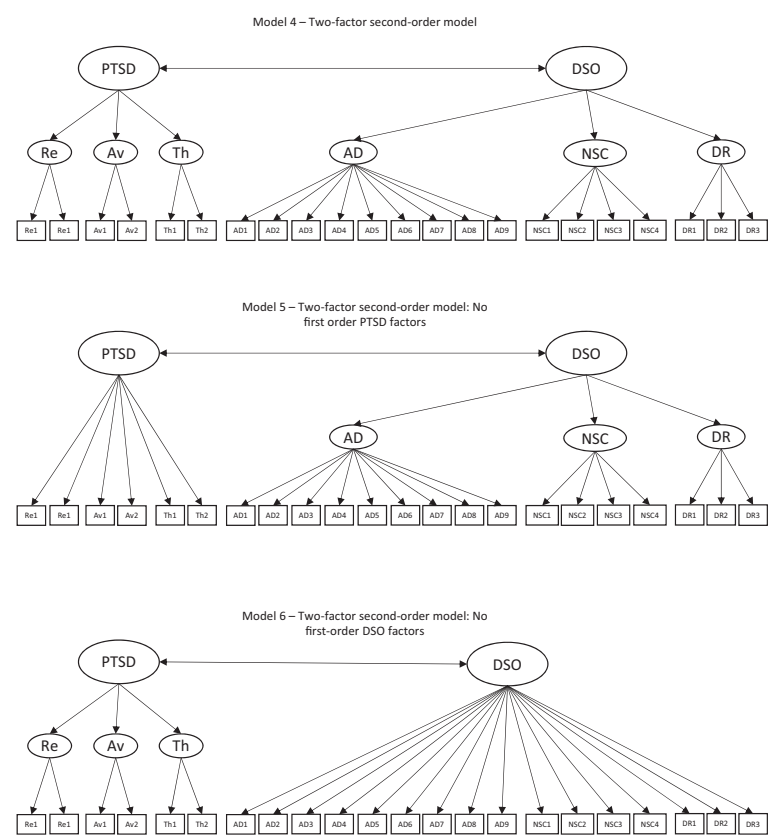

Fig. 1. Alternative models of the latent structure of Complex PTSD symptoms. 
Table 1. Frequencies of symptom endorsement for each PTSD and DSO item of the ITQ, and symptom-based diagnostic estimates

\begin{tabular}{lcc}
\hline & Valid \% (n) & Valid \% (n) \\
\hline & PTSD Dx & CPTSD Dx \\
& $10.9(18)$ & $53.6(89)$ \\
& & \\
PTSD symptom endorsement & $72.7(13)$ & $92.1(82)$ \\
Upsetting dreams (Re1) & $77.8(14)$ & $93.3(83)$ \\
Reliving the event in the here and now (Re2) & $100(18)$ & $95.5(85)$ \\
Upset upon reminders (Re3) & (n) & $100(89)$ \\
Internal avoidance (Av1) & $94.4(17)$ & $93.3(83)$ \\
External avoidance (Av2) & $100(18)$ & $97.8(87)$ \\
Being on guard (Th1) & $88.9(16)$ & $92.1(82)$ \\
Jumpy/startled (Th2) & $72.2(13)$ & \\
DSO symptom endorsement & & \\
Intense reactions (AD1) & $72.2(13)$ & $88.8(79)$ \\
Long time to calm down (AD2) & $72.2(13)$ & $95.5(85)$ \\
Feelings easily hurt (AD3) & $55.6(10)$ & $92.0(81)$ \\
Uncontrollable anger (AD4) & $33.3(6)$ & $58.4(52)$ \\
Reckless behaviour (AD5) & $27.8(5)$ & $48.9(43)$ \\
Numb (AD6) & $44.4(8)$ & $91.0(81)$ \\
Difficulty feeling pleasure (AD7) & $27.8(5)$ & $86.4(76)$ \\
World is distant (AD8) & $83.3(15)$ & $92.1(82)$ \\
Feeling outside of body (AD9) & $61.1(11)$ & $69.3(61)$ \\
Failure (NSC1) & $22.2(4)$ & $85.4(76)$ \\
Worthless (NSC2) & $16.7(3)$ & $80.9(72)$ \\
Self-shame (NSC3) & $33.3(6)$ & $88.8(79)$ \\
Guilt (NSC4) & $33.3(6)$ & $95.5(85)$ \\
Cut-off from others (DR1) & $44.4(8)$ & $96.6(86)$ \\
Difficult to stay close to others (DR2) & $16.7(3)$ & $86.5(77)$ \\
Avoiding relationships (DR3) & $16.7(3)$ & $73.0(65)$ \\
\hline
\end{tabular}

Re, re-experiencing; $A v$, avoidance; Th, sense of current threat; $A D$, affective dysregulation; NSC, negative self-concept; DR, disturbances in relationships; DSO, disturbances in self-organization; PTSD Dx, probable ICD-11 post-traumatic stress disorder diagnostic rates; CPTSD Dx, probable ICD-11 complex PTSD diagnostic rates.

*Probable PTSD and CPTSD diagnostic rates are estimated without the inclusion of Re3.

Factorial validity and composite reliability

Model fit statistics for the ITQ are presented in Table 2. Models 2, 3, and 4 offered the best representations of the factor structure of the ITQ. Inspection of the BIC results indicates that Model 4 offers the optimal representation of the sample data. Model 4, which is consistent with the ICD-11 proposals for PTSD and CPTSD, possessed a chisquare-to-degree of freedom ratio of $<3: 1$ indicating acceptable model fit; CFI and TLI values of .95 indicating excellent fit; however, the RMSEA value was above the criteria for acceptable model fit. Considering all indices together, Model 4 appears to offer a viable representation of the latent structure of the ITQ.

The first- and second-order PTSD factor loadings were all positive, statistically significant $(P<.001)$, and high $(>.70)$. The first- and secondorder DSO factor loadings were all positive, and statistically significant $(P<.001)$. While most firstorder factor loadings were of a robust magnitude, two $\mathrm{AD}$ items (uncontrollable anger and reckless
Table 2. Model fit statistics for the alternative models of the ITO

\begin{tabular}{lccccccc}
\hline Models & $\chi^{2}$ & $d f$ & $P$ & CFI & TLI & RMSEA (90\% CI) & BIC \\
\hline 1 & 1135 & 209 & .000 & .871 & .857 & $.161(.152-.170)$ & 11652 \\
2 & 574 & 194 & .000 & .947 & .937 & $.107(.097-.117)$ & 11237 \\
3 & 554 & 203 & .000 & .951 & .944 & $.101(.091-.111)$ & 11212 \\
$\mathbf{4}$ & $\mathbf{5 5 0}$ & $\mathbf{2 0 2}$ & $\mathbf{. 0 0 0}$ & $\mathbf{. 9 5 2}$ &. $\mathbf{9 4 5}$ & $\mathbf{. 1 0 0}(.090-.111)$ & $\mathbf{1 1 2 0 6}$ \\
5 & 659 & 205 & .000 & .937 & .929 & $.114(.104-.124)$ & 11325 \\
6 & 860 & 205 & .000 & .909 & .897 & $.137(.127-.146)$ & 11439 \\
7 & 947 & 208 & .000 & .897 & .886 & $.144(.135-.154)$ & 11558 \\
\hline
\end{tabular}

Estimator $=$ WLSMV; $n=171 ; \chi^{2}=$ Chi-square Goodness of Fit statistic; $d f=$ degrees of freedom; $P=$ Statistical significance; $\mathrm{CF}$, Comparative Fit Index; TLI, Tucker Lewis Index; RMSEA $(90 \% \mathrm{CI})=$ Root-Mean-Square Error of Approximation with $90 \%$ confidence intervals; $\mathrm{BIC}=$ Bayesian Information Criterion; Best fitting model in bold.

behaviour) possessed factor loadings <.60. Additionally, the standardized factor loading of AD on the second-order DSO factor was 1.00 indicating perfect reliability; a result that often occurs in the context of high levels of multicollinearity but is not outside the normal range of results (34) (see Table 3 for full results). The factor correlation between PTSD and DSO was high $(r=.89$, $P<.001)$.

Composite reliability findings, based on estimates derived from the CFA analysis, indicated that the ITQ possesses excellent internal reliability. The six-first-order subscales demonstrated high levels of reliability $(\mathrm{Re}=.90, \mathrm{Av}=.90, \mathrm{Th}=.86$, $\mathrm{AD}=.92, \mathrm{NSC}=.96$, and $\mathrm{DR}=.90)$, as did the 6 PTSD (.96) and 16 DSO (.97) items.

\section{Discriminant validity analysis}

Hierarchical multiple regression analyzes are presented in Table 4. The sociodemographic variables entered at Step 1 significantly contributed to the explanation of three of the six criterion variables, and the most robust predictor of each outcome was unemployment status. The introduction of the PTSD and DSO variables at Step 2 significantly increased the proportion of variance explained in every criterion variable. The largest effect was for negative trauma-related beliefs about the self $\left(\Delta R^{2}=.49 ; \quad F(2,133)=102.94, \quad P<.001\right)$, followed by symptoms of GAD $\left(\Delta R^{2}=.43 ; F(2\right.$, 149) $=63.04, P<.001)$, depression $\left(\Delta R^{2}=.42 ; F\right.$ $(2,148)=75.21, \quad P<.001)$, negative traumarelated beliefs about the world $\left(\Delta R^{2}=.34 ; F(2\right.$, $149)=50.28, \quad P<.001), \quad$ panic disorder $\left(\Delta R^{2}=.22 ; F(2,149)=22.05, P<.001\right)$, and distress tolerance $\left(\Delta R^{2}=.20 ; \quad F(2,149)=20.33\right.$, $P<.001)$.

PTSD uniquely predicted PD symptoms $(\beta=.40$ $(95 \% \mathrm{CI}=.20, .59), P<.001)$ and was the strongest predictor of GAD symptoms $(\beta=.42(95 \%$ 
Validity of ICD-11 PTSD and CPTSD

Table 3. Standardized factor loadings (standard errors) for Model 4

\begin{tabular}{|c|c|c|c|c|c|c|}
\hline Items & $\operatorname{Re}$ & Av & Th & $A D$ & NSC & DR \\
\hline Upsetting dreams (Re1) & $.83(.04)$ & & & & & \\
\hline Reliving the event in the here and now (Re2) & $.92(.03)$ & & & & & \\
\hline Internal avoidance (Av1) & & $.93(.03)$ & & & & \\
\hline External avoidance (Av2) & & $.89(.04)$ & & & & \\
\hline Being on guard (Th1) & & & $.88(.04)$ & & & \\
\hline Jumpy/Startled (Th2) & & & $.85(.04)$ & & & \\
\hline Intense reactions (AD1) & & & & $.71(.04)$ & & \\
\hline Long time to calm down (AD2) & & & & $.75(.04)$ & & \\
\hline Feelings easily hurt (AD3) & & & & $.64(.05)$ & & \\
\hline Uncontrollable anger (AD4) & & & & $.51(.06)$ & & \\
\hline Reckless behaviour (AD5) & & & & $.56(.06)$ & & \\
\hline Numb (AD6) & & & & $.85(.03)$ & & \\
\hline Difficulty feeling pleasure (AD7) & & & & $.83(.03)$ & & \\
\hline World is distant (AD8) & & & & $.80(.03)$ & & \\
\hline Feeling outside of body (AD9) & & & & $.72(.04)$ & & \\
\hline Failure (NSC1) & & & & & $.95(.01)$ & \\
\hline Worthless (NSC2) & & & & & $.97(.01)$ & \\
\hline Self-shame (NSC3) & & & & & $.90(.02)$ & \\
\hline Guilt (NSC4) & & & & & $.86(.03)$ & \\
\hline Cut-off from others (DR1) & & & & & & $.96(.03)$ \\
\hline Difficult to stay close to others (DR2) & & & & & & $.82(.04)$ \\
\hline Avoiding relationships (DR3) & & & & & & $.79(.04)$ \\
\hline Second-order factor loadings & & & PTSD & & DSO & \\
\hline Re-experiencing (Re) & & & $.81(.04)$ & & & \\
\hline Avoidance (Av) & & & $.71(.04)$ & & & \\
\hline Sense of current threat (Th) & & & $.83(.05)$ & & & \\
\hline Affective dysregulation (AD) & & & & & $1.00(.02)^{*}$ & \\
\hline Negative self-concept (NSC) & & & & & $.74(.04)$ & \\
\hline Disturbances in relationships (DR) & & & & & $.86(.03)$ & \\
\hline
\end{tabular}

All factor loadings are statistically significant $(P<.001)$

Re, re-experiencing in the here and now; Av, avoidance; Th, sense of current threat; AD, affective dysregulation; NSC, negative self-concept; DR, disturbed relationships. *Error variance was 0 indicating perfect reliability.

$\mathrm{CI}=.25, .58), P<.001)$. DSO significantly predicted negative trauma-related beliefs about the self $(\beta=.70(95 \% \mathrm{CI}=.56, .84), P<.001)$, depression $(\beta=.61(95 \% \mathrm{CI}=.46, .76), P<.001)$, negative trauma-related beliefs about the world $(\beta=.53$ $(95 \% \mathrm{CI}=.36, .70), P<.001)$, distress tolerance $(\beta=-.52(95 \% \mathrm{CI}=-.72,-.32), P<.001)$, and GAD symptoms $(\beta=.35(95 \% \quad \mathrm{CI}=.18, .51)$, $P<.001)$.

\section{Discussion}

Consistent with previous findings (2-4), the prevalence of the proposed PTSD and CPTSD diagnoses combined were significantly lower than DSM-5 PTSD. Current and past findings suggest that the revised model of psychotraumatology outlined for ICD-11 provides a stricter criterion for diagnosis than that provided by the DSM-5. While the two systems demonstrated a reasonably high level of agreement regarding who should receive a diagnosis, there was a meaningful subset of individuals who qualified for a diagnosis of PTSD under DSM-5 but did not qualify for a diagnosis of PTSD or CPTSD under ICD-11. In contrast, only one person qualified for a diagnosis of PTSD or CPTSD under ICD-11 but did not qualify for a diagnosis of PTSD under DSM-5.

When the non-specific Re3 symptom was introduced, the combined ICD-11 PTSD and CPTSD diagnostic rates were no longer significantly different from DSM-5. Previous studies using secondary data sources have also found that differences in diagnostic rates between the two manuals are attributable to fewer individuals meeting the ICD-11's Re requirements, and that if at least one non-specific re-experiencing symptom is introduced (e.g., upset upon reminders of the trauma, or, intrusive memories of the trauma), differences in diagnostic rates become non-significant (35). The conceptualization of Re in ICD-11 is unique in that it focuses on the experience of reliving the traumatic event again in the here and now. This emphasis is based on evidence demonstrating that intrusive recollections of traumatic life events are common across psychiatric disorders (36); that re-experiencing in the here and now distinguishes intrusive cognitive processes in PTSD from those observed in other disorders (37). 


\section{Hyland et al.}

Table 4. Standardized factor loadings (standard errors) for Model

\begin{tabular}{|c|c|c|c|c|c|c|}
\hline & Panic & GAD & Depression & $\mathrm{PTCl}$ - self & PTCl - world & Distress tolerance \\
\hline Step $1 R^{2}$ & .04 & .06 & $.16^{* * *}$ & $.20^{* * *}$ & $.15^{* * *}$ & .05 \\
\hline Gender & .05 & -.02 & .00 & .03 & -.01 & -.11 \\
\hline Age & -.11 & .04 & .06 & -.11 & -.14 & .10 \\
\hline Unemployment status & $.17^{*}$ & $.18^{*}$ & $.32^{* * *}$ & $.36^{* * *}$ & $.33^{* * *}$ & -.10 \\
\hline Relationship Status & .05 & .11 & $.16^{*}$ & $.20^{*}$ & .14 & -.11 \\
\hline Step $2 R^{2}$ change & $.22^{* * *}$ & $.43^{* * *}$ & $.42^{* * *}$ & $.49 * * *$ & $.34^{* * *}$ & $.20^{* * *}$ \\
\hline Age & -.08 & .08 & .09 & -.08 & -.11 & .08 \\
\hline Unemployment status & .05 & -.02 & .11 & $.13^{*}$ & $.14^{*}$ & .04 \\
\hline Relationship status & -.05 & -.03 & .03 & .07 & .03 & -.04 \\
\hline PTSD & $.40^{* * *}$ & $.42^{* * *}$ & .13 & .08 & .13 & .06 \\
\hline DSO & .14 & $.35^{* * *}$ & $.61^{* * *}$ & $.70^{* * *}$ & $.53^{* * *}$ & $-.52^{* * *}$ \\
\hline Total variance explained & $26.1 \% * * *$ & $49.0 \% * * *$ & $58.3 \% * * *$ & $68.4 \% * * *$ & $49.3 \% * * *$ & $25.4 \% * * *$ \\
\hline
\end{tabular}

DSO, disturbances in self-organization; PTSD, post-traumatic stress disorder; GAD, generalized anxiety disorder; PTCI - self, negative cognitions of the self-subscale from the Post-traumatic Cognitions Inventory; PTCl - world, negative cognitions about the world-subscale from the Post-traumatic Cognitions Inventory $* P<.05, * * * P<.001$.

The inclusion of a third, non-specific Re symptom is currently under consideration for those who do not possess a clear memory of their index trauma. Only two of 20 individuals who indicated no clear memory of their index trauma lost a diagnosis if this item was excluded; one lost a diagnosis of PTSD, and one lost a diagnosis of CPTSD. Current results suggest that the introduction of a third Re symptom may not be necessary to capture those without a clear memory of their index trauma; however, further research with larger and more diverse trauma samples is necessary to determine the necessity of this third Re symptom.

The CFA findings indicated that the latent structure of the ITQ was best represented by a two-factor second-order model that reflects the ICD-11's distinction between PTSD and DSO symptomatology. This result replicates the only other assessment of the latent structure of the ITQ (11) and is consistent with findings derived from archival data (6-9). The CFA findings partially support the study's second hypothesis: Models 2 and 4, which discriminate between PTSD and DSO symptoms at the first- and second-order level, respectively, evidenced acceptable model fit. However, Model 3 which posits a single higher-order CPTSD factor also evidenced acceptable fit. The generally good fit of Model 2 suggests that while the two-factor second-order model is plausible, and desirable due to its increased parsimony, it is not the only viable factorial solution of the ITQ that captures the distinction between PTSD and DSO symptoms. With respect to Model 3, given that most the sample met diagnostic status for CPTSD $(53.6 \%)$ rather than PTSD $(10.9 \%)$, it is unsurprising that this model offered reasonable fit. It is probable that future studies utilizing clinical samples characterized by high levels of CPTSD will find acceptable fit for this conceptualization. Nonetheless, it was notable that Model 4, which distinguishes between the second-order factors of PTSD and DSO, was deemed to be the most satisfactory representation of the latent structure of the ITQ, despite the strong correlation between these factors. The CFA results, therefore, support a distinction between PTSD and DSO symptomatology among this clinical sample.

The validity of this distinction between PTSD and DSO was further evidenced by the results of the hierarchical multiple regression analyzes. Consistent with the study's third hypothesis, PTSD symptoms, but not DSO symptoms, positively predicted levels of PD; whereas DSO symptoms, but not PTSD symptoms, positively predicted symptoms of depression, and negative cognitions about the self and the world, and negatively predicted distress tolerance scores. PTSD and DSO were both significant, positive predictors of GAD symptoms, but PTSD was a stronger predictor than DSO. While the existing literature has indicated that CPTSD can be meaningfully distinguished from PTSD due to exposure to childhood traumatization (12), chronic traumatization (38), increased psychological distress (13), and female sex (39), the current findings demonstrate that PTSD and DSO possess differentially associated with multiple, clinically relevant criterion variables. These differential associations, the robust magnitude of the effects, and the substantial variance explained in each criterion variable provide substantial empirical support for the discriminant validity of ICD-11 PTSD and CPTSD.

The current study contains several limitations. First, although the sample was typical of secondary and tertiary care clinical groups, the small sample size and clinical nature of the sample limit generalizability to the wider trauma population. 
Replication using larger clinical, and community, samples is needed. Second, given that ICD-11 is still under development and not scheduled for publication until 2018, the ITQ will undergo revisions in the near future. These revisions will focus on streamlining the current set of indicators by which to correspond to the ICD-11 diagnostic guidelines. One of the major organizing principles of the ICD11 is to use as small a number of symptom indicators as possible so as to improve clinical utility (1); therefore, an immediate challenge will center on reducing the current list of DSO symptoms. While current results support the validity and reliability of the ITQ, considerable work will be required to finalize the scale and establish its psychometric properties. Third, although the current study represents the first instance in which DSM-5 and ICD-11 PTSD/CPTSD diagnostic rates have been compared using diagnostic-specific measures, it will be important to replicate the current study using clinician-administered diagnostic scales. Additionally, the current study did not include a measure of functional impairment for DSM-5 and ICD-11 meaning that estimated prevalence rates may be overestimated.

The current study supports the factorial and discriminant validity of ICD-11 PTSD and CPTSD and provides empirical support for the reliability and validity of the newly developed ITQ (10). These findings support the distinction between PTSD and DSO symptoms, and reveal that these constructs possess unique relationships with clinically relevant outcomes. The continued empirical support for ICD-11 CPTSD should encourage clinicians to screen for DSO symptomatology, and emphasizes the need for treatment interventions that are specifically tailored to address these symptoms (40). Concerns have been expressed about the availability of two diagnostic systems that produce discrepant diagnostic rates, particularly for patients and carers as it is possible that one system may be used over another for the purposes of litigation, insurance coverage, and benefit refusal (41). Although these potentially negative consequences are issues that we believe clinicians and researchers should be acutely aware of, they may unfortunately be unavoidable consequences of our continuing search for the most accurate understanding of trauma-related psychopathology.

\section{Declarations of interest}

Chris Brewin and Marylène Cloitre participated as members of the World Health Organization Working Group on the Classification of Disorders Specifically Associated with Stress, reporting to the International Advisory Group for the
Revision of ICD-10 Mental and Behavioural Disorders. However, the views expressed reflect the opinions of the authors and not necessarily the Working Group or Advisory Group and the content of this article does not represent WHO policy. Marylene Cloitre, Neil Roberts, Jonathan Bisson, and Chris Brewin are co-authors of the ITQ. Philip Hyland, Mark Shevlin, Anthony J. Downes, Sandra Jumbe, Thanos Karatzias have no conflicts of interest.

\section{References}

1. Maercker A, Brewin CR, Bryant RA et al. Proposals for mental disorders specifically associated with stress in the ICD-11. Lancet 2013;381:1683-1685.

2. Hansen M, Hyland P, Armour C, Elklit A. Shevlin, M Less is more? Assessing the validity of the ICD-11 model of PTSD across multiple trauma samples. Eur J Psychotraumatol 2015;6:28766.

3. O'Donnell ML, Alkemade N, Nickerson A et al. Impact of the diagnostic changes to post-traumatic stress disorder for DSM-5 and the proposed changes to ICD-11. Br J Psychiatry 2014;205:230-235.

4. Wisco BE, Miller MW, Wolf EJ et al. The impact of proposed changes to ICD-11 on estimates of PTSD prevalence and comorbidity. Psychiatry Res 2016;240:226-233.

5. Hyland P, Brewin C, Maercker A. Predictive validity of ICD-11 PTSD as measured by the Impact-of-Event Scale Revised: a 15-year prospective, longitudinal study of political prisoners. J Trauma Stress 30:125-132.

6. Tay AK, Rees S, Chen J, Kareth M, Silove D. The structure of post-traumatic stress disorder and complex post-traumatic stress disorder amongst West Papuan refugees. BMC Psychiat 2015;15:111.

7. Hyland P, Shevlin M, Elklit A et al. An assessment of the construct validity of the ICD-11 proposals for complex posttraumatic stress disorder. Psychol Trauma 2017;9:1-9.

8. Nickerson A, Cloitre M, Bryant RA, Schnyder U, Morina $\mathrm{N}$, Schick M. The factor structure of complex posttraumatic stress disorder in traumatized refugees. Eur J Psychotraumatol 2016;7:33253.

9. Shevlin M, Hyland P, Karatzias T et al. Alternative models of disorders of traumatic stress based on the new ICD-11 proposals. Acta Psychiat Scand 2017;135:419-428.

10. Cloitre M, Roberts NP, Bisson JI, Brewin CR. The International Trauma Questionnaire (ITQ). Unpublished measure.

11. Karatzias T, Shevlin M, Fyvie $\mathrm{C}$ et al. An initial psychometric assessment of an ICD-11 based measure of PTSD and complex PTSD (ICD-TQ): evidence of construct validity. J Anxiety Disord 2016;44:73-79.

12. Karatzias T, Shevlin M, Fyvie $\mathrm{C}$ et al. Evidence of distinct profiles of Posttraumatic Stress Disorder (PTSD) and Complex Posttraumatic Stress Disorder (CPTSD) based on the new ICD-11 Trauma Questionnaire (ICD-TQ). J Affect Disord 2017;207:181-187.

13. Murphy S, Elklit A, Dokkedahl S, Shevlin M. Testing the validity of the proposed ICD-11 PTSD and complex PTSD criteria using a sample from Northern Uganda. Eur J Psychotraumatol 2015;7:32678.

14. Weathers FW, Litz BT, Keane TM, Palmieri PA, Marx BP, SCHNURR PP. The PTSD checklist for DSM-5 (PCL5), 2013. Instrument available from the National Center for PTSD at www.ptsd.va.gov

15. Weathers FW, Blake DD, Schnurr PP, Kaloupek DG, Marx BP, KeAne TM. The Life Events Checklist for DSM5 (LEC-5), 2013. Instrument available from the National Center for PTSD at www.ptsd.va.gov 


\section{Hyland et al.}

16. Konecky B, Meyer EC, Kimbrel NA, Morrisette SB. The structure of DSM-5 posttraumatic stress disorder symptoms in war veterans. Anxiety Stress Coping 2016;29:497506.

17. Spitzer RL, Kroenke K, Williams JB, Lowe B. A brief measure for assessing generalized anxiety disorder: the GAD7. Arch Intern Med 2006;166:1092-1097.

18. Kertz S, Bigda-Peyton J, Björgvinsson T. Validity of the Generalized Anxiety Disorder-7 scale in an acute psychiatric sample. Clin Psychol Psychother 2013;20:454-464.

19. Kroenke K, Spitzer RL, Williams JBW. The PHQ-9: validity of a brief depression severity measure. J Gen Intern Med 2001;16:606-613.

20. Manea L, Gilbody S, McMillan D. A diagnostic meta-analysis of the Patient Health Questionnaire-9 (PHQ-9) algorithm scoring method as a screen for depression. Gen Hosp Psychiatry 2015;37:67-75.

21. Wittkampf KA, BaAs KD, van Weert HC, Lucassen P, SchENE AH. The psychometric properties of the panic disorder module of the patient health questionnaire (PHQ-PD) in high-risk groups in primary care. J Affect Disord 2011;130:260-267.

22. Foa EB, Ehlers A, Clark DM, Tolin DF, Orsillo SM. The Posttraumatic Cognitions Inventory (PTCI): development and validation. Psychol. Assessment 1999;11:303-314.

23. Simons JS, Gaher RM. The Distress Tolerance Scale: development and validation of a self-report measure. Motiv Emot 2005;29:83-102.

24. Raykos BC, Byrne SM, Watson H. Confirmatory and exploratory factor analysis of the distress tolerance scale (DTS) in a clinical sample of eating disorder patients. Eat Behav 2009;10:215-219.

25. Landis JR, Косн GG. The measurement of observer agreement for categorical data. Biometrics 1977;33:159-174.

26. Muthén LK, MuthéN BO. MPlus user's guide (7th edn). Los Angeles, CA: Muthén \& Muthén; 2013.

27. Flora DB, Curran PJ. An empirical evaluation of alternative methods of estimation for confirmatory factor analysis with ordinal data. Psychol Methods 2004;9: 466-491.

28. Kuine RB. Principles and Practice of Structural Equation Modeling (4th edn). New York, NY: Guilford Press; 2011.
29. BentLer PM. Comparative fit indexes in structural models. Psychol Bull 1990;107:238-246.

30. Steiger JH. Structural model evaluation and modification: an interval estimation approach. Multivariate Behav Res 1990;25:173-180.

31. Yuan KH, Bentler PM. Three likelihood-based methods for mean and covariance structure analysis with nonnormal missing data. Sociol Methodol 2000;30:165-200.

32. RAFTERY AE. Bayesian model selection in social research. Sociol Methodol 1995;25:111-163.

33. RAYKOV T. Estimation of composite reliability for congeneric measures. Appl Psychol Meas 1997;21:173-184.

34. DeEgan J. On the occurrence of standardized regression coefficients greater than one. Educ Psychol Meas 1978;38:873-888.

35. Hyland P, Shevlin M, McNally S, Murphy J, Hansen M, ElKLIT A. Exploring differences between the ICD-11 and DSM-5 models of PTSD: does it matter which model is used? J Anxiety Disord 2016;37:48-53.

36. Brewin CR, Gregory JD, Lipton M, Burgess N. Intrusive images in psychological disorders: characteristics, neural mechanisms, and treatment implications. Psychol Rev 2010;117:210-232.

37. BREWIN CR. Episodic memory, perceptual memory, and their interaction: foundations for a theory of posttraumatic stress disorder. Psychol Bull 2014;140:69-97.

38. Cloitre M, Garvert DW, Brewin CR, Bryant RA, MaerCKER A. Evidence for proposed ICD-11 PTSD and complex PTSD: a latent profile analysis. Eur J Psychotraumatol 2013;4:20706.

39. Perkonigg A, Hofler M, Cloitre M, Wittchen HU, Trautmann S, Maercker A. Evidence for two different ICD-11 posttraumatic stress disorder in a community sample of adolescents and young adults. Eur Arch Psychiatry Clin Neurosci 2016;266:317-328.

40. Cloitre M, Courtois CA, Charuvastra A, Carapezza R, Stolbach BC, GReen BL. Treatment of complex PTSD: results of the ISTSS expert clinician survey on best practices. J Trauma Stress 2011;24:615-627.

41. Bisson JI. What happened to harmonization of the PTSD diagnosis? The divergence of ICD11 and DSM5. Epidemiol Psychiatr Sci 2013;22:205-207. 